\title{
Study on Status of Implementation of Leisure Sports Activities in Huaxi National-level Wetland Park in Guiyang
}

\author{
Zhanfeng Wang \\ School of Physical Education of Guizhou Education University, Guiyang Guizhou, 550018, China
}

Key words: Huaxi National-level Wetland Park, leisure sports activities, Status exploration and analysis.

\begin{abstract}
Huaxi National-level Wetland Park in Guiyang is the most appropriate site for leisure sports. This paper studies the status of implementation of leisure sports activities in Huaxi Wetland Park with research methods such as document literature, expert interview and mathematical statistics. The result shows that physical and mental pleasure and body building are the main purpose of the implementation of leisure sports activities by people in Huaxi Weland Park. Walking and cycling are main leisure sports activities implemented in Huaxi Wetland Park. However, only over ten leisure sports activities have been implemented in the wetland park covering $2.19 \mathrm{~km}^{2}$. Young and middle-aged people are main groups carrying out leisure sports activities in Huaxi Wetland Park and people participating in leisure sports activities frequently are middle-aged and elderly people and adolescent college students. Main factors restricting the implementation of leisure sports activities in Huaxi Wetland Park are the lack of supporting measures such as policy system, weak cultural atmosphere of leisure sports and inadequacy of professional field and facilities. Therefore, this paper puts forward such countermeasures as comprehensive development of leisure sports and characteristic tourism, the improvement of construction of basic conditions required for the implementation of leisure sports activities, advocation of leisure sports activities and theoretical construction so as to promote the implementation of leisure sports activities in Huaxi National-level Wetland Park.
\end{abstract}

\section{Introduction}

Huaxi National Urban Wetland Park located in the north of central urban area of Huaxi District, Guiyang City, only 12KM away from the downtown of Guiyang City, is a national rare urban wetland park. As an open public place of leisure sports in the urban area of Guiyang, Huaxi National Wetland Park in Guiyang is a modernized high-end ecological park integrating culture, nature, play, entertainment and festival activities. With the gradual improvement of living standard and fitness consciousness of people in Guiyang in recent years, the participation in leisure sports exercise has become an important constituent part of the life of people. Huaxi National Wetland Park just provides an important site for the participation of people in leisure sports exercise. Therefore, this paper has important practical significance for promoting the implementation of leisure sports activities in Huaxi National-level Wetland Park and impelling people to attend leisure sports activities positively through investigation on the status of implementation of leisure sports activities in Huaxi Wetland Park. 


\section{Analysis on status of implementation of leisure sports activities in Huaxi Wetland Park}

\section{Analysis on purpose and motivation of leisure sports activities in Huaxi Wetland Park}

Good purpose and motivation are the motive power of people to conduct leisure sports. Correct motivation of people has important significance for participating in leisure sports activities and obtaining good experience. Positive participation in leisure sports activities has important functions for forming good social attitude, establishing good interpersonal relationship, improving team awareness and promoting the progress of social civilization. According to study, the main purpose of people for participating in leisure sports activities in Huaxi National-level Wetland Park is physical and mental pleasure and physical exercise. A small number of people attend leisure sports activities mainly for killing time and strengthening interpersonal communication. With the improvement of material living standard of people, participation in leisure sports activities mainly for the purpose of physical and mental pleasure and body building has become a major part of the daily life of people. The two purposes account for $65.5 \%$ and $46 \%$ of the total number of people participating in leisure sports. In addition, people participating in leisure sports activities can not only improve their emotion and adjust spirit, but also make friends by participating in leisure sports activities in Huaxi Wetland Park.

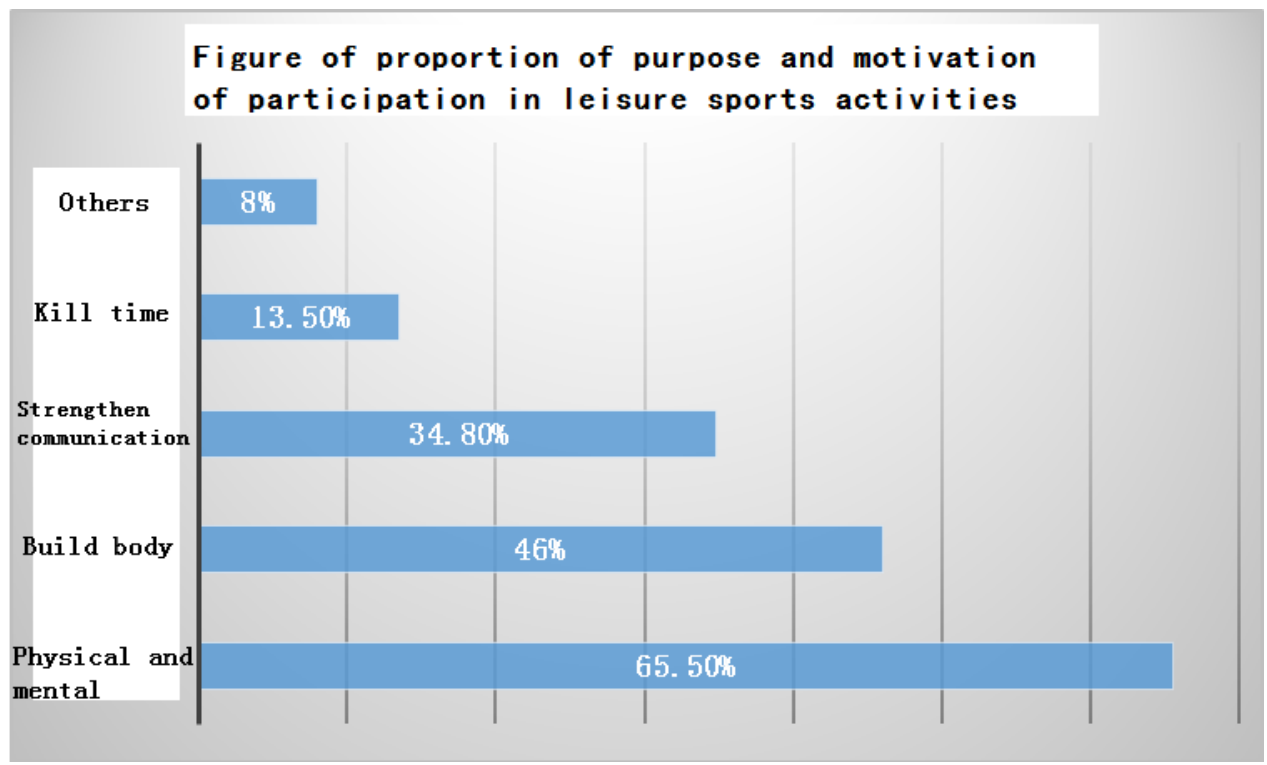

Fig. 1. Distribution of motivation of people for participating in leisure sports activities in Huaxi Wetland Park

\section{Analysis on situation of implementation of leisure sports activities in Huaxi Wetland Park}

Leisure sports activity program is the generic terms of various activity contents and methods by means of body movement for the purpose of physical and mental pleasure, body building and the improvement of sports technique level. Huaxi Wetland Park is the most appropriate place for taking leisure sports exercise. Leisure sports activities implemented currently mainly include walking, cycling, running, Taichi for health, social dance, square dancing, fishing, boating, spinning top beating and chess playing. According to statistical analysis on the number of people participating in these events, people walking in Huaxi Wetland Park account for $73 \%$ of the total number under investigation. The reason is that walking is a simple exercise with low strength which can be done at any time in any place for a long time. Meanwhile, Huaxi Wetland Park is near the mountain and by the river with many vegetations and beautiful natural environment. Many nearby residents in Guiyang go to Huaxi Wetland Park at leisure time for sightseeing and work and study pressure regulation. Cycling ranks second among leisure sports activities, accounting for $38.2 \%$ of the total number of people under investigation. The main reason is that the gathering place of various universities in Guiyang is near Huaxi Wetland Park. Many adolescent students often cycle to Huaxi Wetland Park at spare time for sightseeing, fitness and play. Cycling of a group of vital adolescent 
students in Huaxi Wetland Park becomes a distinct and beautiful scenery. Leisure sports events such as running, Taichi for health, social dance, square dancing, fitness dance, fishing, spinning top beating, boating and chess playing are mainly activities implemented by residents near Huaxi Wetland Park for leisure and recreation and body building. Group events such as social dance, square dancing and fitness dance are mainly implemented in the morning and evening every day, which last for 1 to 3 hours each time, accounting for 25\%.Though there are over ten leisure sports activities implemented in Huaxi Wetland Park, it seems to be a small number in the park 6.5km long and covering an area of 2.19

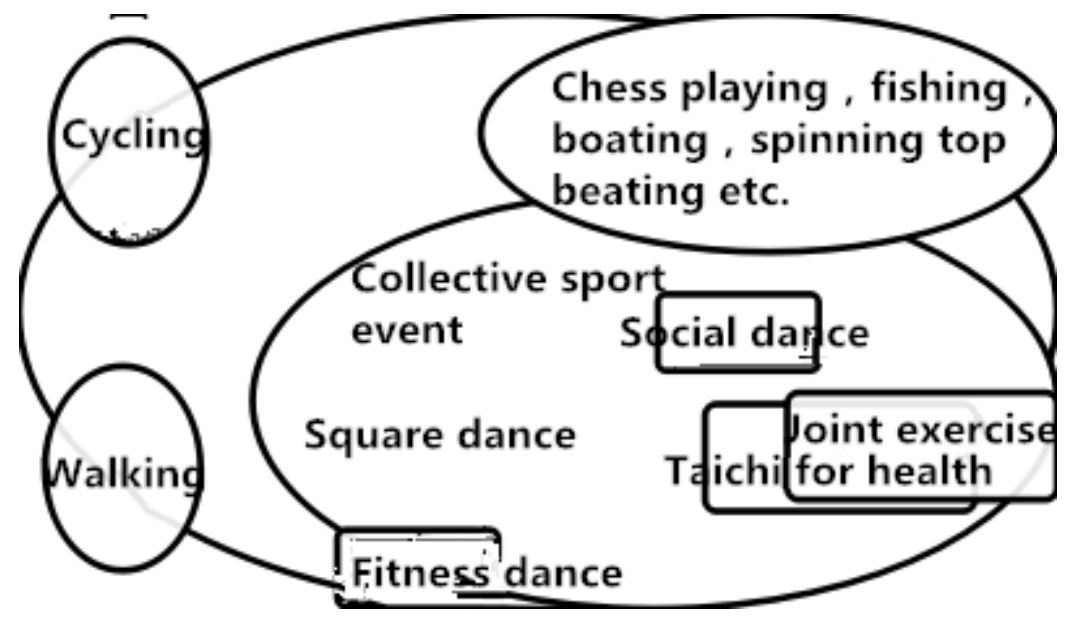

Fig. 2. Distribution of events of people for participating in leisure sports activities in Huaxi Wetland Park

\section{Analysis on features of people participating in leisure sports activities in Huaxi Wetland Park}

Analysis on gender feature of people participating in leisure sports activities in Huaxi Wetland Park Gender structure of people participating in leisure sports activities can reflect the relationship between the purpose of people for physical exercise and the event. Proportions of males and females participating in leisure sports activities in Huaxi Wetland Park have great differences. There are obviously more females than males. The main reason is that such events as square dancing, social dance, fitness dance and walking do not have high requirements for activity space and the basis and age of participating objectives and are easy to learn, organize and implement. Females prefer to such rhythmic leisure sports events. Therefore, there are more females than males participating in leisure sports activities in the park. Male participators prefer to exciting sports events. There are few exciting leisure sports events except cycling. Therefore, few males participate.

Analysis on age structure of people participating in leisure sports activities in Huaxi Wetland Park People taking leisure sports exercise in Huaxi Wetland Park are mainly young and middle-aged people. Teenagers under 20 account for 7.6\%; young people between 20 and 29, 32.7\%; middle-aged people between 29 and 45, 26\%; middle-aged and elderly people between 46 and 60, 19.3\%; elderly people above $60,14.4 \%$.According to the distribution of their age structure, age feature of people participating in leisure sports activities in the park is characterized by "a large portion in the middle and a small portion at both ends" . The reason is that people in the age group between 20 and 45 have fully realized the advantage of leisure sports activities. Moreover, due to universities and business district around the park, there are many people at the age level.

\section{Analysis on factors of implementation of leisure sports activities in Huaxi Wetland Park}

Lack of policy system promoting implementation of leisure sports activities in Huaxi Wetland Park Policy system is the premise guaranteeing leisure sports activities in Huaxi Wetland Park. The administrative department of Huaxi Wetland Park and relevant institution can guarantee the time, space and field and equipment required for leisure sports activities in Huaxi Wetland Park only by establishing complete policy system for promoting the implementation of leisure sports activities in the park. According to investigation, Huaxi Wetland Park has not established relevant policy system 
and rules and regulations for leisure sports activities, which is not good for the implementation of leisure sports activities in the park.

Cultural construction of leisure sports activities to be strengthened

Good cultural atmosphere of leisure sports has important functions for improving the concept and awareness of people about sports activities. According to study, no one in Huaxi National Wetland Park has conducted management and construction about leisure sports culture. There are only some sports and health formulas and slogans on the health footpath. There is no relevant publicity of sports culture in other places. Few relevant organizations and units implement leisure sports activities in Huaxi Wetland Park. Only a fitness footpath and several fitness slogans cannot produce enough influence on people. Therefore, weak leisure sports culture in Huaxi National-level Wetland Park has become one of the main factors restricting the implementation of leisure sports activities.

Lack of site and equipment required for implementation of leisure sports activities

Site and equipment of leisure sports activities are the guarantee of basic conditions for the participation of people in leisure sports activities as well as main contents of cultural construction of leisure sports. Though Huaxi Wetland Park covers a large area, most areas in the park are forest, flower nursery, pond, brook and mountain for enjoyment, some undeveloped areas, sidewalk road, square and open space. There are few professional site and equipment required for the implementation of leisure sports activities. With diversified demands of people for contents of leisure sports activities, the lack of professional site and equipment is not good for leisure sports activities in Huaxi Wetland Park. The addition of some leisure sports facilities and site appropriate for interpersonal communication and exchange in Huaxi Wetland Park, such as children's amusement park, basketball court, badminton hall and table tennis hall which are sports events favored by the public, can promote exchange and realize the effect of fitness. People will be more willing to spend more time in leisure sports activities in the park.

\section{Suggestions}

\section{Create comprehensive development of leisure sports and characteristic tourism}

Leisure sports is an important branch in modern tourism system, which has attracted a lot of sports travel enthusiasts at home and abroad. Huaxi Wetland Park is an urban wetland park with karst features. Its unique geographic position and geologic structure form rich and diversified scenery and geographical resources. Therefore, creating a development path of Huaxi Wetland Park with the combination of leisure sports and characteristic tourism with advantaged resources is an important way for leading the development of leisure sports well. Park leisure sports activities are gradually influencing us. Development with the combination of characteristic tourism and leisure sports represents the general trend.

\section{Strengthening construction of basic conditions required for implementation of leisure sports activities}

To promote the implementation of leisure sports activities in Huaxi National-level Wetland Park, on the one hand, relevant administrative department and organizations should promote the implementation of leisure sports activities by establishing relevant policy system and supporting measures. On the other hand, they should improve and enrich infrastructures such as site and equipment required for the implementation of leisure sports activities through special funds input. Moreover, leisure sports cultural construction in Huaxi Wetland Park should be enriched with multiple approaches and methods. For example, the implementation of leisure sports activities in Huaxi National-level Wetland Park can be promoted through the establishment of a special institution responsible for promoting the implementation of leisure sports activities and a special fitness area for leisure sports activities and frequent implementation of sports cultural activities. 


\section{Advocate leisure sports activities and strengthen theoretical construction}

With rapid development of economy in recent years, the demand of people for leisure sports has constantly increased. Vigorous development of leisure sports has become a key point of national social sports work. In particular, the integration of slow motion has promoted the development of leisure sports. However, under the situation of general increase of social pressure, spare time is occupied by excessive working time; the awareness of people about leisure sports is suppressed and they cannot pursue for comfortable life state. Finally, advocating leisure sports activities and updating the concept and consciousness can facilitate physical and mental relaxation of people and promote the development of leisure sports activities. It is required to strengthen theoretical study on leisure sports activities in Huaxi Wetland Park with multiple approaches and methods under the background of dissatisfactory implementation of leisure sports activities in the park so as to make leisure sports activities a beautiful scenery and feature in Huaxi Wetland Park.

\section{Acknowledgement}

Fund program: educational scientific planning issue of Guizhou in 2014 (2014C017).

\section{References}

[1] Yao Chunqing, Peng Chun. Economic Analysis and Prospect of Development of Leisure Sports Industry in China, Youthful Days, 2016, (5). 258.

[2] Xu Fenglian, Gao Qianjin, Fei Mingguo et al. Investigation on Physical Fitness Status of Community Residents in Shijiazhuang, Journal of Shijiazhuang University, 2008 (3): 92-95.

[3] Li Peng, Gao Liang. Investigation on Status of Participation of Residents in Jiaozuo in Leisure Sports Activities and Study on Countermeasures, Liaoning Sport Science and Technology, 2016,(1):37-41.

[4] Li Xiaoqi, Guo Song. Investigation and Analysis on Implementation of Leisure Sports Activities in Urban Areas of Guiyang, Guizhou Sports Science and Technology, 2007 (4): 29-33.

[5] Tang Yi. Investigation on Leisure Sports in Huichuan, Zunyi, Space Time of Sport, 2016, (Volume 2). 36-37.

[6] Sun Fenglin. Diachronic and Ecological Investigation and Analysis on Leisure Sports in a Park, Sports Science, 2015,(5).16-28.

[7] Wang Junqi, Cheng Yali, Zhang Haiyang. Analysis on Participation of the Elderly in Park Leisure Sports Activities, Urban Problem, 2012,09:74-77.

[8] Tang Shaokang. Status Investigation and Countermeasure Study on Leisure Sports Activities in Urban Riverside Park, Fujian Normal University, 2015. 